\title{
Social support and the psychological wellbeing of people living with HIV/AIDS in Ghana
}

\section{K Oppong Asante}

Department of Human Development and Psychology, Regent University College of Science and Technology, Accra, Ghana

\begin{abstract}
Objective: The current study sought to investigate the association between age, gender, social support and the psychological wellbeing of people living with HIV and AIDS (PLHA) in Ghana. Method: Cross-sectional data containing information on demographics, social support and psychological well-being (stress, depression, and anxiety), were collected from 107 men and women living with HIV/AIDS. To explore age group differences, participants were stratified by age (<39 vs. $40+$ years). Three anonymous self-administered questionnaires were used, namely the demographic data questionnaire, Sources of Social Support Scale, and the Depression Anxiety Stress Scale (DASS- 42). Results: Correlation analysis revealed that social support was negatively associated with depression, stress and anxiety. Compared with males living with HIV, women reported higher levels of stress, depression and anxiety. Female gender and low social support were significant predictors of depression and stress after controlling for selected independent variables. Older participants experienced higher levels of stress than their younger counterparts. Conclusion: Public health personnel and AIDS professionals may consider further interventions to promote psychological health in HIV/AIDS-positive individuals. More attention should be paid to the social environment of individuals diagnosed with HIV as the quality of social relationships may be particularly important for successful psychological adaptation to HIV.
\end{abstract}

Keywords: HIV/AIDS; Psychological wellbeing; Social support; Ghana

Received: 11-08-2011

Accepted: $12-09-2011$

doi: http://dx.doi.org/10.4314/ajpsy.v15i5.42

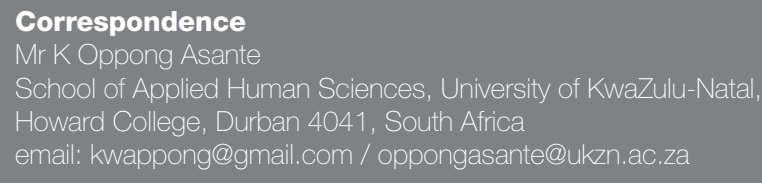

email: kwappong@gmail.com / oppongasante@ukzn.ac.za

\section{Introduction}

HIV and AIDS is one of the most complicated and bewildering social challenges faced by contemporary societies due to its strong ties with sexual and societal stigmatized behavior. Contracting HIV may lead to difficulties related to self-esteem, coping, social isolation, and poor psychological well-being. ${ }^{1,2}$ By the end of 2009, 33. ${ }^{3}$ million people worldwide were living with HIV; 2.6 million people became newly infected and 1.8 million people had lost their lives to AIDS in the same year. 3 With the alarming increase in the HIV/AIDS pandemic in developing countries, and the limited accessibility and availability of highly active antiretroviral therapy (HAART),
\end{abstract}

the majority of people living with HIV/AIDS (PLHA) continue to suffer with the disease, with a serious impact on their well-being. 4,5

A considerable amount of research have been conducted in many countries on the variables related to the psychological well-being of different populations of PLHA. ${ }^{6-}$ ${ }_{10}$ Current research confirms that receiving social support from significant social network members can promote positive psychological adjustment in people living with HIV. Greater amounts of social support have been shown to be associated with less negative and more positive affect in people living with HIV and AIDS. ${ }^{11-13}$ Moreover, people living with HIV and AIDS who are satisfied with the amount of support available to them tend to experience less psychological distress, a higher quality of life, and more self-esteem ${ }^{7,14-16}$ whereas those who perceive low levels of social support experience increased distress. ${ }^{6}$

Researchers suggest that sexual orientation influences the association between social support and psychological 
well-being among people living with HIV/AIDS. ${ }^{13}$ Women living with HIV/AIDS experience lower quality of life, fewer social supports and more depressed than men living with HIV/AIDS. ${ }^{17}$

Another important socio-demographic correlate of psychological well-being is age. Studies suggest that older adults may fare better and be more psychologically resilient than their younger counterparts. Comparisons of younger and older adults with HIV/AIDS, older adults experience either comparable or fewer depressive symptoms and greater overall emotional well-being than younger age groups. ${ }^{18,19}$ Some plausible explanations that have been offered for these age differences include patience and contentment in old age, less threatening perceptions of illness, less resentment due to being diagnosed with a chronic condition, and greater wisdom. ${ }^{20}$

Despite evidence for the association between agerelated changes in social support and enhanced psychological well-being, the literature on this association among individuals with HIV is sparse. Moreover, less is known about how men and women living with HIV/AIDS may differentially perceive and integrate support into their lives, and how this support subsequently explains their psychological well-being. The relationships among social support and psychological wellbeing among PLHA in Ghana have not been well established.

To address these gaps in the literature, the present study examines how support from partners and family/friends is associated with psychological wellbeing in a Ghanaian sample of men and women living with HIV and AIDS, and also to ascertain whether older adults would report of fewer depressive symptoms and more positive affect than their younger counterparts. Empirical evidence about the relationships between these factors will provide better understanding of the challenges facing PLHA in Ghana and set scientific foundations for future intervention development.

\section{Method \\ Sample}

The sample in the study comprised of 107 out-patients living with HIV/AIDS who were receiving medical treatment at the Fevers' Unit of the Korle-Bu Teaching Hospital, Accra, Ghana. Participants were made up of 39 males (36.4\%) and 68 females (63.6\%) with an age range of 18 to 58 years. The sample size represented $97 \%$ response rate from eligible prospective individuals approached to take part in the study. The purposive sampling was used to select the health facility where People Living with HIV/AIDS (PLHAs) receive medication from Opportunistic Infections and the convenience sampling was used to select the participants for the study. All the participants met the following inclusion criteria: diagnosed as HIV+, at least 18 years of age and cognitively intact.

\section{Measures}

Social support: The Sources of Social Support Scale ${ }^{21}$ was used to assess participants' perceptions of the psychosocial support they received from family/friends, church members and from partners. Prior research finds the internal consistencies of the subscales to be satisfactory, with a
Cronbach's alpha of .73.21 The Sources of Social Support Scale had been validated with a Ghanaian sample which yielded a Cronbach's alpha coefficient of 0.78 indicating that the instrument could be used in a Ghanaian context. ${ }^{22}$ In this study, the Cronbach's alpha ( $\alpha$ ) coefficient was .81 for the Sources of Social Support Scale.The reliability of the subscale were judged sufficient because the alpha value was well above $.60^{23}$ which is the minimum requirement. The subscale consisted of 10 items (e.g., financial assistance, give you reassurance, encouragement and emotional support, show affection, etc) and participants indicated whether they receive psychosocial support a lot to not receiving support at all on a score range of 1-5. Items were summed to create the score for each participant with a maximum score of 50 and a least score of 10.

Psychological wellbeing: Psychological well-being was measured using the 42-item self report of the Depression Anxiety Stress Scale (DASS- 42). ${ }^{24}$ The DASS-42 is a well structured questionnaire consisting of three subscales of 14 items each measures stress, depression, and anxiety. The depression subscale assesses dysphoria, hopelessness, devaluation of life, self-depreciation, amotivation and anhedonia. The anxiety subscale assesses autonomic arousal, situational anxiety and subjective experience of anxiety. The stress subscale assesses difficulty relaxing, nervousness, agitation and irritability. ${ }^{25}$ The internal consistencies of the three subscales were 0.71 for depression, 0.79 for anxiety and 0.81 for stress. The overall validity and reliability of DASS-42 have been well established. ${ }^{25-27}$ The DASS-42 has a score range of 0 (does not apply to me at all) to 3 (applied to me very much, or most of the time). The Cronbach's $\alpha$ coefficient for the DASS -42 was .74 in this study. The cut-off values for depression subscale was (0-9, 10-13, 14-20, 21-27, and 28+); anxiety subscale was (0-7, 8-9, 10-14, 15-19, and 20+), whilst the stress subscale was (0-14, 15-18, 19-25, 26-33, and 34+). The above mentioned cut-off represents five the categories (normal, mild, moderate, severe and extremely severe) on the subscales. The percentages of participants falling into each of the five categories are reported in Table II.

\section{Procedure}

Data was collected from study site, using anonymous self reported questionnaires with validated and reliable scales. Each subject was administered the DASS-42 and the Sources of Social Support Scale (SSSS) to complete. Initial screenings of PLHA were conducted in the hospitals and performed by healthcare workers and research staff specifically hired for the study. Once the PLHA had been screened and had agreed to participate in the study, written informed consent was obtained.

Following informed consent, each participant was given the anonymous self reported questionnaire and asked to fill them. The questionnaire, which was in English language elicited information about their demographics, including age, gender, educational status, and questions about their perceived social support, depression, stress and anxiety. It took an average of 45 minutes to complete both questionnaires, and the whole data was collected within a period of 2 weeks. A total of 115 individuals voluntarily 
participated in the assessment. Refusal rate was approximately 7\%. Because of multiple missing data points, 107 participants are included in this current study.

\section{Ethical consideration}

Participants were assured that the data will be used for research purpose, and that participation was voluntary. Approval of this study was obtained from Institutional Review Boards from the Ghana Health Service Ethical Review Committee, under the Ministry of Health, and the Human Research Committee of Regent University College of Science \& Technology.

\section{Data Analysis/ Strategy}

All analyses were performed using the Statistical Package for the Social Sciences (SPSS Inc, Chicago 18.0 version) was used for data entry, validation and analysis. First, descriptive statistics were used to describe PLHA's demographics, perceived social support and the level of general psychological status. Second, Pearson correlation coefficients were calculated to examine the relationship between stress, anxiety, depression and social support, as well as demographic variables such gender, age, and education. Third, a series of multiple regression analyses were conducted to examine the associations of age and social support on the indicators of psychological wellbeing (depression, anxiety and stress) among PLHA. Regression coefficients estimation and their significance levels are reported.

\section{Results}

Table I shows the characteristics of PLHAs in the study. The average of the participants was 37.2 years $(\mathrm{SD}=9.50$ ). Majority of the sample, $75.7 \%$ received higher education, $91.6 \%$ had contracted HIV through sexual contact and the remainder contracted it through blood transfusion (1.9\%), or an unknown route of transmission (6.5\%). The mean scores of social support, depression, anxiety and stress were $22.66(\mathrm{SD}=7.53), 18.74(\mathrm{SD}=4.60), 9.95(\mathrm{SD}=2.39)$ and $15.72(\mathrm{SD}=3.22)$ respectively

The means, SDs and ranges for each of the three DASS sub-scales are presented in Table II for the total sample. Additionally, for each subscale the percentage of participants falling into each of the five categories (normal, mild, moderate, severe and extremely severe) created by the use of cut-off scores ${ }^{25}$ is presented. However, these cut-offs have been presented purely for comparative purposes, and it is important to reiterate that

\begin{tabular}{|l|l|l|}
\hline \multicolumn{3}{|l|}{ Table I: Demographic data of the participants (n = 107) } \\
\hline Variables & N & $\%$ \\
\hline Ages (yrs) & & \\
$18-39$ (young) & 59 & 55.1 \\
$40+$ (old) & 48 & 44.9 \\
\hline Gender & & \\
Males & 39 & 36.4 \\
Females & 68 & 63.6 \\
\hline Marital Status & & \\
Unmarried & 39 & 36.4 \\
Married & 88 & 46.7 \\
Divorced & 2 & 1.9 \\
Widowed & 16 & 15.0 \\
\hline Educational Level & & \\
Higher education & 81 & 75.7 \\
Lower education & 26 & 24.3 \\
\hline Mechanism of contracting HIV & & \\
Sexual contact & 98 & 91.6 \\
Blood transfusion & 2 & 1.9 \\
Unknown & 7 & 6.5 \\
\hline
\end{tabular}

DASS scores should be regarded as providing an individual's score on an underlying dimension.

The correlation coefficients among demographic characteristics, social support, depression, anxiety and stress are shown in Table III. It was observed that the level of depression experienced by PLHA.s was significantly correlated with the levels of anxiety $(r=0.68, p<0.01)$, and unsurprisingly, significantly negatively correlated with social support $(r=-0.44, p<0.01)$. Social support received correlated negatively with the amount of stress experienced $(r=-0.15, p=0.090)$ and significantly negative correlated with the levels of anxiety $(r=-0.52, p$ $<0.01$ )

Significant correlations were also found among PLHA's demographic characteristics and depression, anxiety, stress and social support. Being female was significantly correlated depression $(r=0.83, p<0.01)$, anxiety $(r=$ $0.83, p<0.01)$, and stress $(r=0.22, p=0.02)$, but significant correlated negatively with social support (being male), $(r=-0.63, p<0.01)$. Age of PLHA (young or old) was significantly correlated negatively with social

Table II: Summary statistics for the DASS-42 ( $\mathrm{n}=107)$

\begin{tabular}{|c|c|c|c|c|c|c|c|c|}
\hline & & & & \multicolumn{5}{|c|}{ Percentage in each DASS category } \\
\hline & $M$ & $S D$ & Range & Normal & Mild & Moderate & Severe & Extremely Severe \\
\hline Depression & 17.4 & 4.60 & $0-28$ & 20.8 & 17.4 & 30.1 & 29.3 & 2.4 \\
\hline Anxiety & 15.07 & 4.70 & $0-26$ & 24.7 & 12.1 & 28.5 & 23.9 & 10.8 \\
\hline Stress & 15.77 & 3.21 & $0-36$ & 31.8 & 34.8 & 21.1 & 9.4 & 2.9 \\
\hline
\end{tabular}




\begin{tabular}{|c|c|c|c|c|c|c|}
\hline Source & 1 & 2 & 3 & 4 & 5 & 6 \\
\hline \multicolumn{7}{|l|}{ 1. Gender } \\
\hline 2. Age & .06 & & & & & \\
\hline 3. Social support & $-.63^{\star *}$ & $-.20^{\star}$ & & & & \\
\hline 4. Depression & $.83^{\star \star}$ & .01 & $-.44^{\star \star}$ & & & \\
\hline 5. Anxiety & $.83^{\star \star}$ & .09 & $-.52^{\star \star}$ & $.68^{*}$ & & \\
\hline 6. Stress & $.22^{*}$ & .11 & -.17 & .12 & .15 & \\
\hline
\end{tabular}

support $(r=-0.20, p<0.05)$ in favour of the young, and correlated with the amount of stress ( $r=0.11, p=0.247$ ), in favour of old PLHA. Education was significantly correlated with levels of stress $(r=0.27, p<0.01)$.

Table IV shows the multiple regression models examining factors associated with depression, anxiety and stress. Controlling for selected independent variables, higher levels of social support was significantly associated with lower levels of depression (standardized beta $(\beta)=$ $.18, \mathrm{p}<0.0001)$, stress $(\beta)=.37, \mathrm{p}<0.0001)$ but not for anxiety $(\beta=.07, \mathrm{p}=.024)$, for both men and women living HIV. Together, social support from family members accounted for $5.4 \%$ of the variance in depression ( $\mathrm{p}<$ 005), $1.1 \%$ for stress $(p<005)$, and $7.2 \%$ for anxiety ( $p<$ $001)$.

In the regression model, older participants experienced higher levels of stress that their younger counterparts $(\beta=.17, \mathrm{p}<0.001)$. In addition, for women living with HIV, lower levels of social support was associated with higher levels of anxiety $(\beta=.96, p<0.05)$, depression $(\beta=.88, \mathrm{p}<0.05)$, and stress $(\beta=.37, \mathrm{p}<$ $0.0001)$

\section{Discussion}

This study aimed to establish the association between social support and the psychological wellbeing of people living with HIV and AIDS (PLHA) in Ghana. Consistent with prior research ${ }^{11-13,17}$, women living with HIV in our study experienced higher rates of stress, depression and anxiety than their male counterparts, and received less social support from their friends and families. Social support was expected to be beneficial for both men and women living with HIV. However, the findings indicated that social support was instrumental in determining the psychological well-being of men and women living with HIV. Social support was also associated with gender, such that men living with HIV derived more psychological benefits than women living with HIV. The results also indicated that social support may have positive implication for the psychological well-being of men and women living with HIV.

A plausible explanation could be that self-esteem may have played a role in the extent to which women were able to perceive and receive support efforts from friends and families. Self-esteem has been shown to have an influence

\begin{tabular}{|c|c|c|c|c|c|c|}
\hline \multirow[t]{2}{*}{ Variables } & \multicolumn{2}{|c|}{ Depression } & \multicolumn{2}{|c|}{ Anxiety } & \multicolumn{2}{|l|}{ Stress } \\
\hline & $\beta$ & $p$-Value & $\beta$ & $p$-Value & $\beta$ & $p$-Value \\
\hline Age & .04 & .068 & .06 & .068 & $.17^{*}$ & .004 \\
\hline Gender & $.88^{\star \star}$ & $<$ & $.96^{\star *}$ & $<.0001$ & $.24^{\star \star}$ & $<.0001$ \\
\hline Social & .0001 & & -.07 & .024 & $-.37^{\star \star}$ & $<.0001$ \\
\hline Support & $-.18^{\star \star}$ & $<$ & .0001 & .072 & .010 & \\
\hline$R^{2}$ & .054 & & & & & \\
\hline
\end{tabular}


on interpersonal relationships because individuals' feelings of self-worth have a bearing on both their beliefs and social behaviors. ${ }^{28}$ This means that low self-esteem may damage interpersonal relationships because it promotes a selfprotective interpersonal style. ${ }^{28}$ In other words, PLHA feel socially isolated and have negative perceptions of their social relationships. This process may be fueled by internalized HIV-related stigma and discrimination, which has been described as an emotional reaction to the many different layers of overt HIV-related stigma people living with HIV have to face. ${ }^{29}$ It can therefore be implied that a lack of positive social relationships leads to negative psychological states such as anxiety or depression, and that only support that is perceived as adequate would influence the appraisal process and function as a stress buffer. ${ }^{30}$

It is also possible that women living with HIV/AIDS in this study did not want to forgo their duties and responsibilities as care provider as a result of their illness. ${ }^{31}$ If family members provided unsolicited important support by offering to help with child care, providing advice, or even offering financial assistance, women may have perceived the message that they were not capable of managing the norms and responsibilities of their social role. Support that is not asked for can be perceived by support recipients as controlling or interfering, and is often met with negative reactions and feelings of incompetence. ${ }^{32}$ In contrast, men whose social roles prescribe that they be recipients of care rather than providers of care ${ }^{33}$ may have seen involvement from family as welcome and helpful, and likely did not feel as if the norms of their social role were being threatened.

It was also expected that older adults would report of fewer depressive symptoms and more positive affect than their younger counterparts. Results indicated that there no significant difference in psychological well-being between age groups. These findings on the psychological well-being between young and older PLHA could suggests that their stress, anxiety and depression levels may be the same. This finding contradicts the summary provided by 18,19 that compared with younger adults with HIV/AIDS, older adults experience either comparable or fewer depressive symptoms and greater overall emotional well-being than younger age groups. There was however, a small negative significant relationship between age between social support, indicating that younger adults received more social support from friends and families that their older counterparts.

Older PLHA differs from younger PLHA in a number of respects. They feel less positive about themselves, have poor physical health and live with a range of medical conditions in addition to AIDS. ${ }^{7}$ It was however, surprisingly that their psychological wellbeing is not significantly different from younger PLHA in Ghana, a finding that has been collaborated in a clinical-based sample. ${ }^{34}$ It could be due to the fact that both the younger and the older PLHA were able to obtain and utilize health care information, and also both groups had accepted their HIV status, thereby overcoming related stigma associated with the disease.

The evidence of age differences in social support could be explained by the socioemotional selectivity theory. ${ }^{35}$ According to this theory; younger people sustain larger networks than older people in order to maximize the information acquisition function of social support. As people age, they become more selective in their social interaction, so that they can "maximize social and emotional gains and minimize social and emotional risks" (p.331). ${ }^{35}$ Hence, older people are more motivated to maintain social relationship with few friends and family with whom they feel emotionally close, and to withdraw from social relationships with mere acquaintances, as these relationships may not be emotionally rewarding.

\section{Study limitations}

There are some limitations to this study. First, the data analyses were done based on a cross - sectional data; therefore, causal interpretation of the results cannot be established. The results cannot be generalized to the entire population of people living with HIV/AIDS in Ghana.

Another limitation of the study was that all variables were measured by self-report instruments, which may have caused some bias. It is important for future studies also to use other forms of data collection, such as interviews or expert judgments. Finally, several aspects that could also be related to psychological well-being, such as coping strategies and personality characteristics were not included in the present study. Future studies should try to include these other issues as well.

\section{Conclusion}

Despite these shortcomings, the present study adds to the existing knowledge between social support and depression, stress as well as anxiety, and also identified some demographic that could influence factors that the psychological wellbeing of PLHA. There are social, economic and cultural issues concerning People Living with HIV/AIDS within this study area. The findings have shown the importance of social support, and other forms of support to PLHA in order to improve their well-being.

The provision of social support structures will enable PLHA to cope better with the disease, which could lead to a sense of satisfaction, fulfillment and hope. This could also lead to the reduction of stigma and discrimination towards PLHA, and encourage an open discussion about HIV and free disclosure of HIV status. It is also important to give PLHA a voice and encourage social inclusion as this could enhance their psychological well-being. The government should also consider factors that could improve the wellbeing of PLHA in addition to the provision of antiretroviral drugs.

Public health personnel and AIDS professionals may consider further interventions to promote psychological health in HIV/AIDS-positive individuals. More attention should be paid to the social environment of individuals diagnosed with HIV as the quality of social relationships may be particularly important for successful psychological adaptation to HIV.

\section{Acknowledgements}

The author is grateful to the participants and the authorities of the Fever's Unit of the Korle-Bu Teaching Hospital for their cooperation. Many thanks to our colleagues who read the draft manuscript and made comments. 


\section{References}

1. Vanable PA, Blair DC, Littelwood RA. Impact of HIV-related stigma on health behaviors and psychological adjustment among HIV-positive men and women. AIDS and Behavior 2006; 10: 473-482.

2. Parker P, Aggleton P, Attawel K, Pulerwitz J, Brown L. HIVIAIDS related stigma and discrimination: a conceptual framework and an agenda for action. Horizons Program. The Population Council. Accessed at: http://www.popcouncil.org/pdfs/horizons/ on 15th June, 2010

3. UNAIDS, \&WHO. AIDS epidemic update 2010. Geneva, Switzerland: UNAIDS. 2010.

4. Leserman J. Role of depression, stress, and trauma in HIV disease progression. Psychosomatic Medicine 2008; 70:539-545.

5. Murri R, Lepri AC, Phillips AN, Girardi E, Nasti G, Ferrara, S., et al. Access to antiretroviral treatment, incidence of sustained therapy interruptions, and risk of clinical events according to sex: Evidence from the I. Co.N.A. Study. Journal of Acquired Immune Deficiency Syndromes 2003; 34(2): 184-190.

6. Catz SL, Gore-Felton C, McClure JB. Psychological distress among minority and low-income women living with HIV. Behavioral Medicine, 2002; 28: 53-60.

7. Turner-Cobb JM, Gore-Felton C, Marouf F, Koopman C, Kim P, Israelski D, et al. Coping, social support, and attachment style as psychosocial correlates of adjustment in men and women with HIVIAIDS. Journal of Behavioral Medicine 2002; 25(4): 337-353.

8. Emlet CA. An examination of the social network and social isolation of older and younger adults living with HIVIAIDS. Health and Social Work 2006; 31:299 - 308.

9. Sun HM, Zhang JJ, Fu XD. Psychological status, coping and social support of people living with HIVIAIDS in Central China. Public Health Nursing 2007; 24:132 - 140.

10. Mavandadi S, Zanjani F, Ten Have TR, Oslin, DW. Psychological well-being among individuals aging with HIV: The value of social relationships. Journal of Acquired Immune Deficiency Syndromes 2009; 51 (2):91 - 98.

11. Deichert NT, Fekete EM, Boarts JM, Druley JA, Delahanty DL. Emotional support and affect: Associations with health behaviors and active coping efforts in men living with HIV. AIDS and Behavior 2008; 12:139-145.

12. Gonzalez JS, Penedo FJ, Antoni MH, Duran RE, Fernandez MI, McPherson-Baker S, et al. Social support, positive states of mind, and HIV treatment adherence in men and women living with HIVIAIDS. Health Psychology 2004; 23:413-418.

13. McDowell TL, Serovich JM. The effect of perceived and actual social support on the mental health of HIV-positive persons. AIDS Care 2007; 19:1223-1229.

14. Safren SA, Radomsky AS, Otto MW, Salomon E. Predictors of psychological well-being in a diverse sample of HIV-positive patients receiving highly active antiretroviral therapy. Psychosomatics 2002; 43:478-485.

15. Johnson AM, Mercer CH, Erens B, Copas AJ, McManus S, Wellings $K$, et al. Sexual behaviour in Britain: partnerships, practices, and HIV risk behaviours. The Lancet 2001; 358:1835 - 1842.

16. Li L, Sung-Jae L, Panithee T, Chuleeporn J, Rotheram-Borus MJ. Stigma, social support and depression among people living with HIV in Thailand. AIDS Care 2009; 21:1007 - 1013.

17. Cederfjäll C, Langius-Eklöf A, Lidman K, Wredling R. Gender differences in perceived health-related quality of life among patients with HIV infection. AIDS Patient Care and STDs 2001; 15:31-39.
18. Crystal S, Akincigil A, Sambamoorthi U, Wenger N, Fleishman J, Zingmond $D$. The diverse older HIV positive population: a national profile of economic circumstances, social support, and quality of life. Journal of Acquired Immune Deficiency Syndrome 2003; 33: $76-83$.

19. Nokes KM, Holzemer WL, Corless IB, Bakken S, Brown MA, Powell-Cop GM. Health-related quality of life in persons younger and older than 50 who are living with HIVIAIDS. Research on Aging 2000; 22: $290-310$.

20. Siegel K, Raveis V, Karus D. Perceived advantages and disadvantages of age among older HIV infected adults. Research on Aging 1998; 20:686 - 711 .

21. Carver CS. Sources of Social Support Scale. Accessed at http://WWW.psy.miami.edu/faculty/ccarver/sclSSSS.html on 2nd Sept., 2010. (2006).

22. Yobanyo E. Social support and its influence on the psychological well-being of expectant mothers in the Accra metropolitan area of Ghana. UnPublished master's thesis in University of Ghana, Legon. (2008).

23. Nunnally JC. Psychometric Theory. New York: 1967.

24. Brown TA, Korotitsch W, Chorpita BF, Barlow DH. Psychometric properties of the Depression Anxiety Stress Scales (DASS) in clinical samples. Behaviour Research and Therapy 1997; 35: 79-89.

25. Lovibond SH, Lovibond PF. Manual for the Depression Anxiety Stress Scales. 2nd Edition: Sydney. 1998.

26. Lovibond PF, Lovibond SH. The structure of negative emotional states: Comparison of the Depression Anxiety Stress Scales (DASS) with the Beck Depression and Anxiety Inventories. Behaviour Research and Therapy 1995); 33: 335-343.

27. Crawford JR, Henry JD. The depression anxiety stress scale (DASS): Normative data and latent structure in a large nonclinical sample. British Journal of Clinical Psychology 2003; 42 : $111-131$.

28. Stinson DA, Logel C, Zanna M, Holmes J, Cameron J, Wood J, et al. The cost of lower self-esteem: Testing a self- and social-bonds model of health. Journal of Personality and Social Psychology 2008; 94: 412-428.

29. Stewart WT., Herek GM, Ramakrishna J, Chandy S, Wrubel J, Elstrand ML O. HIV-related stigma: adapting a theoretical framework for the use in India. Social Science and Medicine 2008; 67: 1225-1235.

30. Cohen S, Wills, TA. Stress, social support, and the buffering hypothesis. Psychological Bulletin 1985; 98: 310 - 357.

31. Revenson TA, Abraído-Lanza AF, Majerovitz SD, Jordan C. Couples coping with chronic illness: What's gender got to do with it? In T. A. Revenson, K. Kayser, \& G. Bodenmann (Eds.), Couples coping with stress: Emerging perspectives on dyadic coping (pp. 137156). Washington, DC: American Psychological Association, 2005.

32. Smith J, Goodnow JJ. Unasked-for support and unsolicited advice: Age and the quality of social experience. Psychology and Aging 1999; 14, 108-121.

33. Eagly AH, Wood W, Deikman AB. Social role theory of sex differences and similarities: A current appraisal. In T. Eckes \& H. M. Trautner (Eds.), The developmental social psychology of gender (pp. 123-174). Mahwah, NJ: Lawrence Erlbaum, 2000.

34. Help Age International. HIVIAIDs and Aging: A brief paper. Accessed at www.helpage.org/publication on 20th April, 2011 2003.

35. Carstensen LL. Social and emotional patterns in adulthood: support for the socioemotional selectivity theory. Psychology and Aging 1992; 7, 331-338. 